\title{
THE VERY YOUNG TYPE Ia SUPERNOVA 2012cg: DISCOVERY AND EARLY-TIME FOLLOW-UP OBSERVATIONS
}

\author{
Jeffrey M. Silverman ${ }^{1}$, Mohan Ganeshalingam ${ }^{1}$, S. Bradley Cenko ${ }^{1}$, Alexei V. Filippenko ${ }^{1}$, \\ Weidong Li ${ }^{1,10}$, Aaron J. Barth ${ }^{2}$, Daniel J. Carson ${ }^{2}$, Michael Childress ${ }^{3}$, Kelsey I. Clubb ${ }^{1}$, \\ Antonino Cucchiara ${ }^{4}$, Melissa L. Graham ${ }^{5}, 6$, G. H. Marion ${ }^{7}$, My L. Nguyen $^{2}$, Liuyi Pei $^{2}$, \\ Brad E. Tucker ${ }^{3}$, Jozsef Vinko ${ }^{8,9}$, J. Craig Wheeler ${ }^{9}$, and Gabor Worseck ${ }^{3}$ \\ ${ }^{1}$ Department of Astronomy, University of California, Berkeley, CA 94720-3411, USA; jsilverman@ astro.berkeley.edu \\ ${ }^{2}$ Department of Physics and Astronomy, University of California, Irvine, CA 92697-4575, USA \\ ${ }^{3}$ The Research School of Astronomy and Astrophysics, The Australian National University, Mount Stromlo and \\ Siding Spring Observatories, Weston Creek, ACT 2611, Australia \\ ${ }^{4}$ Department of Astronomy and Astrophysics, UCO/Lick Observatory, University of California, Santa Cruz, CA 95064, USA \\ ${ }^{5}$ Las Cumbres Observatory Global Telescope Network, Goleta, CA 93117, USA \\ ${ }^{6}$ Department of Physics, Broida Hall, University of California, Santa Barbara, CA 93106, USA \\ ${ }^{7}$ Harvard-Smithsonian Center for Astrophysics, Cambridge, MA 02138, USA \\ ${ }^{8}$ Department of Optics and Quantum Electronics, University of Szeged, Dóm tér 9, H-6720 Szeged, Hungary \\ ${ }^{9}$ Department of Astronomy, University of Texas, Austin, TX 78712-0259, USA \\ Received 2012 June 6; accepted 2012 July 3; published 2012 August 9
}

\begin{abstract}
On 2012 May 17.2 UT, only $1.5 \pm 0.2$ days after explosion, we discovered SN 2012cg, a Type Ia supernova (SN Ia) in NGC $4424(d \approx 15 \mathrm{Mpc}$ ). As a result of the newly modified strategy employed by the Lick Observatory Supernova Search, a sequence of filtered images was obtained starting $161 \mathrm{~s}$ after discovery. Utilizing recent models describing the interaction of supernova (SN) ejecta with a companion star, we rule out a $\sim 1 M_{\odot}$ companion for half of all viewing angles and a red-giant companion for nearly all orientations. SN $2012 \mathrm{cg}$ reached a $B$-band maximum of $12.09 \pm 0.02 \mathrm{mag}$ on 2012 June 2.0 and took $\sim 17.3$ days from explosion to reach this, typical for SNe Ia. Our pre-maximum-brightness photometry shows a narrower-than-average $B$-band light curve for SN $2012 \mathrm{cg}$, though slightly overluminous at maximum brightness and with normal color evolution (including some of the earliest SN Ia filtered photometry ever obtained). Spectral fits to SN 2012cg reveal ions typically found in SNe Ia at early times, with expansion velocities $\gtrsim 14,000 \mathrm{~km} \mathrm{~s}^{-1}$ at 2.5 days past explosion. Absorption from C II is detected early, as well as high-velocity components of both Si II $\lambda 6355$ and Ca II. Our last spectrum (13.5 days past explosion) resembles that of the somewhat peculiar SN Ia 1999aa. This suggests that SN 2012cg will have a slower-than-average declining light curve, which may be surprising given the faster-than-average rising light curve.
\end{abstract}

Key words: supernovae: general - supernovae: individual: SN 2012cg

\section{INTRODUCTION}

Type Ia supernovae ( $\mathrm{SNe}$ Ia) provided the first clear indication that the expansion of the universe is accelerating (Riess et al. 1998; Perlmutter et al. 1999) and have been used as precise distance indicators to accurately measure cosmological parameters (e.g., Conley et al. 2011; Suzuki et al. 2012). Broadly speaking, $\mathrm{SNe}$ Ia are the result of the thermonuclear explosion of $\mathrm{C} / \mathrm{O}$ white dwarfs (WDs); however, the specifics of the progenitor systems and explosion mechanisms are still unclear (see Howell 2011 for further information).

While there is much value in large statistical studies of $\mathrm{SNe}$ Ia, an in-depth investigation of an individual object can be extremely enlightening as well. Two recent examples of this are SN 2009ig and SN 2011fe (PTF11kly). Both are very nearby, normal SNe Ia that were discovered extremely young (Kleiser et al. 2009; Nugent et al. 2011a) and have been intensely studied by many groups and at all wavelengths. These rich data sets are excellent test cases for simulations of SNe Ia and also place strong observational constraints on many theoretical models (e.g., Foley et al. 2012; Bloom et al. 2012; Röpke et al. 2012; Horesh et al. 2012; Parrent et al. 2012).

Here we add to this list a nearby SN Ia that was found 1.5 days after explosion: SN 2012cg. In Section 2 we describe

\footnotetext{
${ }^{10}$ Deceased 2011 December 12.
}

our discovery and present our autonomously triggered follow-up observations, initiated mere minutes after discovery. We present our post-discovery observations and describe our data reduction in Section 3, and our analysis of the pre-maximum-brightness data is discussed in Section 4. We summarize our conclusions in Section 5.

\section{DISCOVERY AND AUTONOMOUS FOLLOW-UP OBSERVATIONS}

As part of the Lick Observatory Supernova Search (LOSS; Filippenko et al. 2001), we have imaged the field of NGC 4424 with the $0.76 \mathrm{~m}$ Katzman Automatic Imaging Telescope (KAIT; Richmond et al. 1993) more than 200 times over the last 14 years. In an $18 \mathrm{~s}$ unfiltered KAIT image beginning at 05:21:26 on 2012 May 17 (UT dates are used throughout), we identified a new transient source with J2000.0 coordinates $\alpha=12^{\mathrm{h}} 27^{\mathrm{m}} 12^{\prime \prime} .83$, $\delta=+09^{\circ} 25^{\prime} 13^{\prime \prime} .1$ (with an uncertainty of 200 mas in each coordinate; Cenko et al. 2012). We found that SN 2012cg had $R=16.92 \pm 0.05 \mathrm{mag}$ at the time of the KAIT discovery (statistical uncertainty only). The next day the object was spectroscopically classified as an extremely young SN Ia (Cenko et al. 2012).

SN 2012cg is located 17". 3 east and 1".5 south of the center of the peculiar SBa galaxy NGC 4424, at a distance of $15.2 \pm$ $1.9 \mathrm{Mpc}$ (from the Tully-Fisher relation; Cortés et al. 2008); 
Table 1

Journal of Spectroscopic Observations of SN 2012cg

\begin{tabular}{lccccc}
\hline \hline UT Date & Epoch $^{\text {a }}$ & Instrument & $\begin{array}{c}\text { Range } \\
(\AA)\end{array}$ & $\begin{array}{c}\text { Resolution }^{\text {b }}(\AA) \\
(\AA)\end{array}$ & $\begin{array}{c}\text { Exposure } \\
(\mathrm{s})\end{array}$ \\
\hline 2012 May 18.234 & 2.5 & Kast & $3434-8198$ & $4.3 / 5.7$ & $2 \times 1500$ \\
2012 May 19.231 & 3.5 & Kast & $3440-8200$ & $4.3 / 5.6$ & $2 \times 1200$ \\
2012 May 20.226 & 4.5 & LRS & $4200-10200$ & 13.5 & 1200 \\
2012 May 20.191 & 4.5 & Kast & $3482-5550$ & 4.4 & 1800 \\
2012 May 21.186 & 5.5 & Kast & $3456-10250$ & $4.4 / 10.9$ & $2 \times 300$ \\
2012 May 22.454 & 6.8 & WiFeS & $3700-9550^{\text {c }}$ & $0.8 / 1.3$ & 600 \\
2012 May 23.368 & 7.7 & WiFeS & $3700-9550^{\text {c }}$ & $0.8 / 1.3$ & 600 \\
2012 May 25.200 & 9.5 & LRS & $4200-10200$ & 13.5 & 900 \\
2012 May 26.235 & 10.5 & Kast & $3490-8314$ & $5.2 / 5.9$ & 300 \\
2012 May 28.198 & 12.5 & Kast & $3490-8124$ & $5.1 / 5.8$ & 300 \\
2012 May 29.180 & 13.5 & Kast & $3452-10232$ & $4.0 / 10.5$ & 13.5 \\
2012 May 29.194 & 13.5 & LRS & $4200-10200$ & 300 \\
\hline
\end{tabular}

Notes.

a Days relative to the date of explosion, 2012 May 15.7. To convert to days relative to $B$-band maximum brightness, subtract the 17.3 day rise time from this number (see Section 4.1 for more information).

b Approximate FWHM intensity resolution. If two numbers are listed, they represent the blue- and red-side resolutions, respectively.

c There is a gap in the spectral coverage at 5574-5630 ̊.

thus, SN 2012cg lies a projected distance of $\sim 1.4 \mathrm{kpc}$ from the host-galaxy center. This places the supernova (SN) just outside a central concentration of $\mathrm{CO}$ and $\mathrm{H} \alpha$ emission, but among many blue stars which may indicate recent (but not ongoing) star formation in the vicinity of SN 2012cg (Cortés et al. 2006).

Beginning in early 2011, we modified the LOSS search strategy with the principal objective of promptly identifying very young (i.e., hours to days after explosion) SNe in nearby galaxies. In addition to decreasing the number of galaxies monitored regularly by KAIT (to increase our observing cadence), we also implemented new software tools to identify SN candidates in nearly real time. Consequently, KAIT autonomously obtained a sequence of $U, B, V$, and unfiltered (roughly $R$ ) images of the transient beginning only $161 \mathrm{~s}$ after the completion of the discovery image. These are some of the earliest filtered photometry data ever obtained of an SN. Subsequent filtered (BVRI) photometry was manually inserted into the KAIT schedule (see Section 3.1).

\section{POST-DISCOVERY OBSERVATIONS AND DATA REDUCTION}

\subsection{Photometry}

KAIT continued to monitor SN $2012 \mathrm{cg}$, and the filtered BVRI follow-up photometry through $\sim 2.5$ weeks after discovery is analyzed herein. These data were reduced using our imagereduction pipeline (Ganeshalingam et al. 2010). Lacking hostgalaxy templates, point-spread function fitting photometry was performed at the position of the SN and seven local comparison stars using the DAOPHOT package in IRAF. ${ }^{11}$ Instrumental magnitudes were transformed to Landolt (1992) system magnitudes using color terms derived from multiple photometric nights. The photometric zero point for each image was determined using the measured magnitudes of the local standards from Sloan Digital Sky Survey transformed into the Landolt standard system (Jordi et al. 2006).

\footnotetext{
11 IRAF is distributed by the National Optical Astronomy Observatory, which is operated by the Association of Universities for Research in Astronomy (AURA) under cooperative agreement with the National Science Foundation (NSF).
}

\subsection{Spectroscopy}

Nearly nightly spectroscopic follow-up observations of SN 2012cg were also initiated after discovery. Low-resolution optical spectra were obtained mainly using the Kast double spectrograph on the Shane $3 \mathrm{~m}$ telescope at Lick Observatory (Miller \& Stone 1993), though data were also acquired with the Marcario Low-Resolution Spectrograph (LRS; Hill et al. 1998) on the $9.2 \mathrm{~m}$ Hobby-Eberly Telescope (HET) at McDonald Observatory and the Wide Field Spectrograph (WiFeS, an integral field unit) on the Australian National University $2.3 \mathrm{~m}$ telescope at Siding Springs Observatory (Dopita et al. 2007). Table 1 summarizes the spectral data of SN 2012cg presented here.

All spectra were reduced using standard techniques (e.g., Silverman et al. 2012a). Routine CCD processing and spectrum extraction were completed with IRAF. We obtained the wavelength scale from low-order polynomial fits to calibration-lamp spectra. Small wavelength shifts were then applied to the data after cross-correlating a template sky to the night-sky lines that were extracted with the SN. Using our own IDL routines, we fit spectrophotometric standard-star spectra to the data in order to flux-calibrate our spectra and to remove telluric lines (Wade \& Horne 1988; Matheson et al. 2000).

\section{ANALYSIS AND RESULTS}

\subsection{Light Curves}

We present our BVRI light curves of SN 2012cg in Figure 1, compared with those of SN 2011fe (unpublished KAIT data), SN 1999aa (Jha et al. 2006), and SN 2005cf (Wang et al. 2009). From a low-order polynomial fit, we find that SN $2012 \mathrm{cg}$ reached a peak $B$-band magnitude of $12.09 \pm 0.02$ on 2012 June $2.0 \pm 0.75$.

Assuming $E(B-V)_{\text {host }}=0.18 \mathrm{mag}$ (see below) and $d=15.2 \mathrm{Mpc}$ (Cortés et al. 2008), this implies that $M_{B}=$ $-19.73 \pm 0.30 \mathrm{mag}$, which is $\sim 0.5 \mathrm{mag}$ brighter than a "normal" SN Ia and $\sim 0.25$ mag brighter than the somewhat peculiar SN 1999aa (Ganeshalingam et al. 2010). However, based on our data through maximum brightness, SN 2012cg 


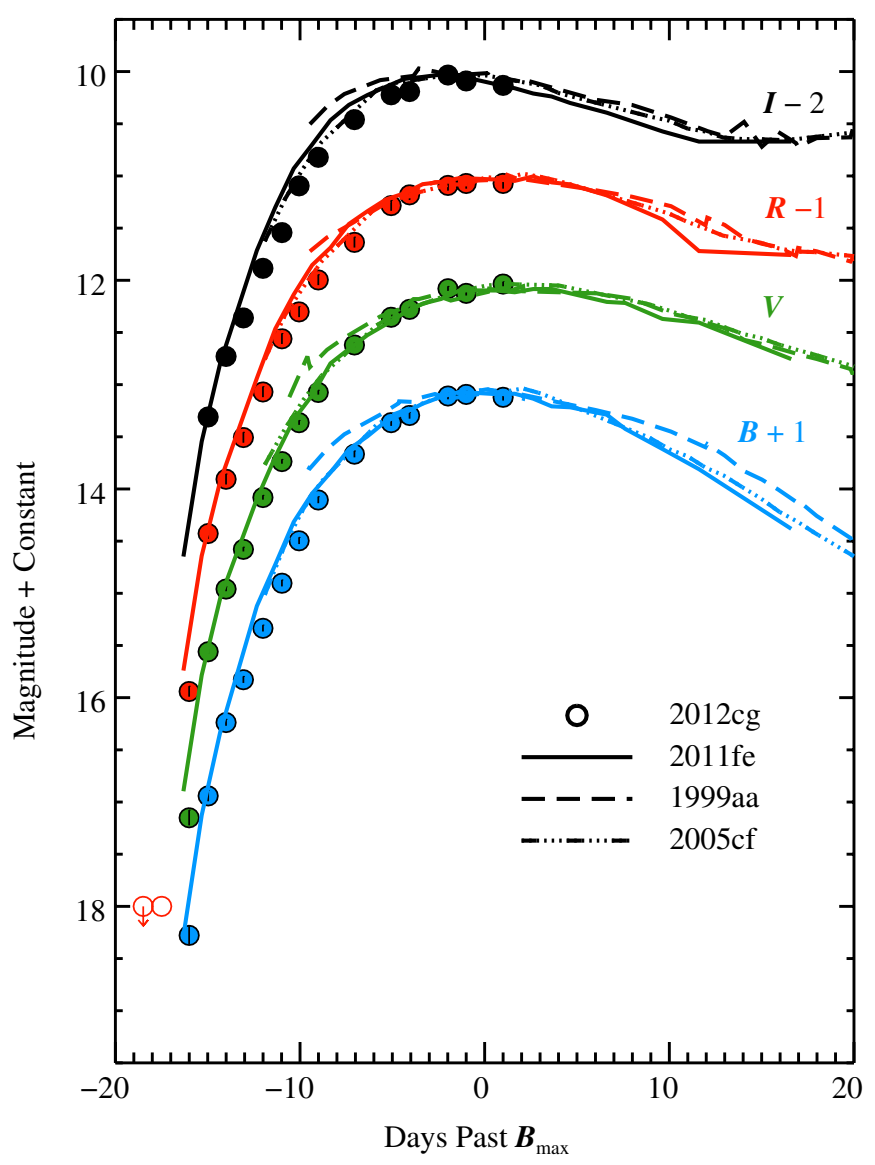

Figure 1. BVRI light curves of SN $2012 \mathrm{cg}$, along with comparisons to $\mathrm{SNe}$ Ia 2011fe, 1999aa, and 2005cf. Comparison light curves have been shifted to have the same peak magnitude and phase as SN 2012cg. Our multi-filter data start within minutes after our discovery image, $\sim 1.5$ days after explosion. A nondetection (upper limit; Cortini 2012) and the pre-discovery detection (open circle; Lipunov \& Krushinsky 2012) are also shown.

appears to have a narrower $B$-band light curve than the "normal" Type Ia SNe $2011 \mathrm{fe}$ and $2005 \mathrm{cf}$ and the slightly overluminous SN 1999aa (even though it resembles this object spectroscopically; see Section 4.2.3).

To determine the date of explosion, we model SN 2012cg as an expanding fireball whose luminosity increases quadratically with time (Riess et al. 1999; Nugent et al. 2011b). Restricting ourselves to the five earliest $B$-band points ( -15.8 to -11.8 days relative to $B$-band maximum brightness), we find that SN $2012 \mathrm{cg}$ was discovered $1.5 \pm 0.2$ days after explosion (which occurred on 2012 May 15.7). This is consistent with nondetections on May $12.3(R>19.8 \mathrm{mag}, \mathrm{KAIT})$ and May 14.9 ( $R>19.0$ mag; Cortini 2012), as well as with the faint "pre-discovery detection" on May $15.8(R \approx 19.0$ mag; Lipunov \& Krushinsky 2012). We measure a rise from explosion to maximum light in the $B$ band of $\sim 17.3$ days, which is within the range of rise times of typical SNe Ia (e.g., Ganeshalingam et al. 2011). We note, however, that our earliest photometry epochs may include a contribution from the host galaxy due to a lack of galaxy templates. A more accurate measurement will be made after galaxy templates are obtained and will likely push our earliest photometric data slightly fainter than presented here.

Shock heating of SN Ia ejecta via interaction with a companion is predicted (Kasen 2010) to produce thermal emission whose temperature and luminosity depend both on the binary separation $(a)$ and the observer angle $(\theta)$. In the case

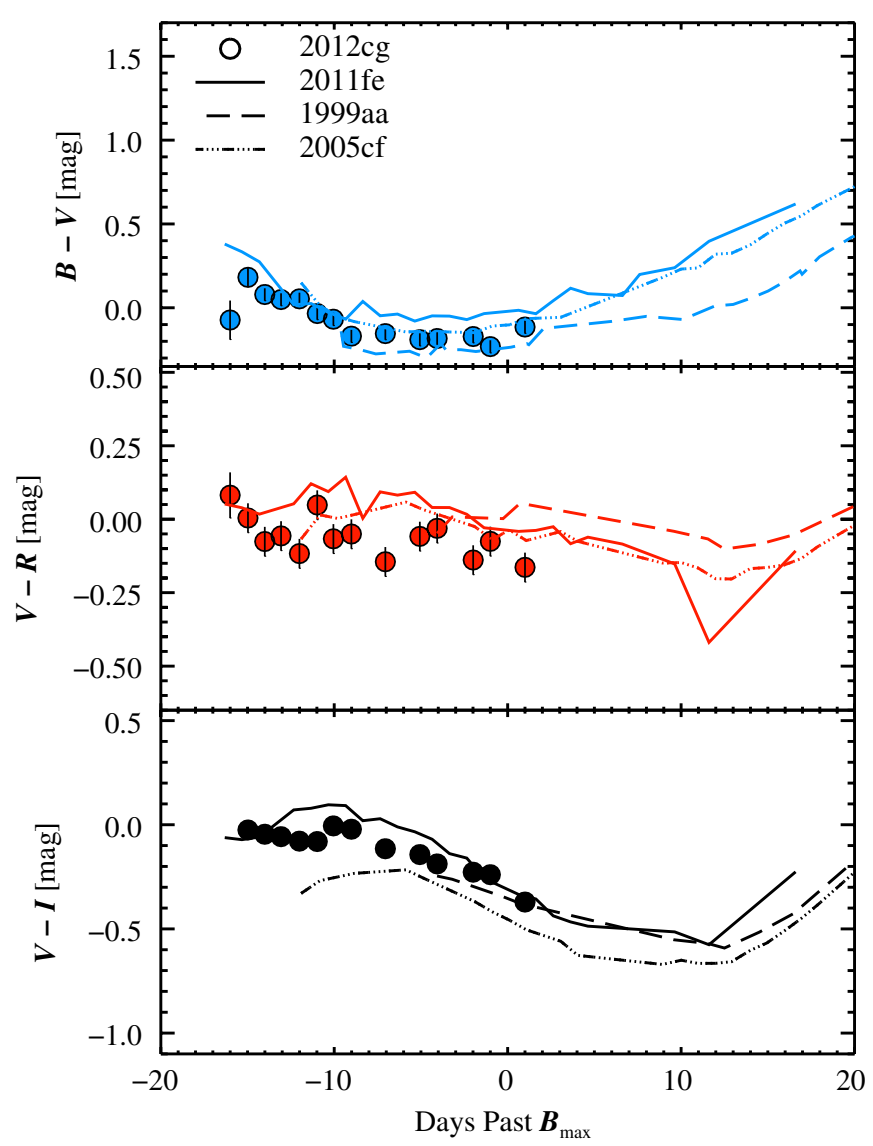

Figure 2. Color curves of SN 2012cg, corrected for $E(B-V)_{\mathrm{MW}}=0.019 \mathrm{mag}$ and $E(B-V)_{\text {host }}=0.18 \mathrm{mag}$. Shown for comparison are SNe 2011fe, 1999aa, and $2005 \mathrm{cf}$, all corrected for extinction using the reddening values provided in their respective references.

of SN $2012 \mathrm{cg}$, the early-time $(t<2$ days) KAIT observations limit any emission from companion interaction with the SN ejecta to $v L_{v}<10^{41} \mathrm{erg} \mathrm{s}^{-1}$ at optical frequencies. Using the analytic models from Kasen (2010), our limits can rule out a $\sim 1 M_{\odot}\left(a \approx 2 R_{\odot}\right)$ main-sequence companion for orientations with $\theta \gtrsim 90^{\circ}$ (i.e., the companion is perpendicular to our line of sight, or slightly farther away than the exploding WD) and red-giant companions ( $a \approx 400 R_{\odot}$ ) for all orientations except when the companion is directly behind the WD. Analytic models of the emission signature of shock breakout (e.g., Chevalier 1992; Rabinak et al. 2011) predict very similar behavior to the (on-axis, or $\theta=0^{\circ}$ ) interaction models of Kasen (2010). From a similar line of reasoning, our early KAIT limits also require that the exploding star must have an initial radius $R_{0} \lesssim 2 R_{\odot}$.

In Figure 2 we plot the evolution of the color of SN 2012cg with time, along with some comparison objects. All objects have been corrected for Galactic extinction using the dust maps of Schlegel et al. (1998) and host-galaxy extinction values from their respective references. No host-galaxy extinction is adopted for SN 2011fe. In order to match our color curves to those of other $\mathrm{SNe}$ Ia, we require $E(B-V)_{\text {host }} \approx 0.18 \mathrm{mag}$. In general, SN 2012cg appears to follow the color evolution of the "normal" Type Ia SNe $2011 \mathrm{fe}$ and 2005cf, as well as the somewhat peculiar SN 1999aa. We also note that these are some of the earliest color data for any SN Ia.

\subsection{Spectra}

Our spectra of SN 2012cg from the first $\sim 2$ weeks are shown in Figure 3. All of the spectra exhibit narrow $\mathrm{Na}$ I D and Ca II 


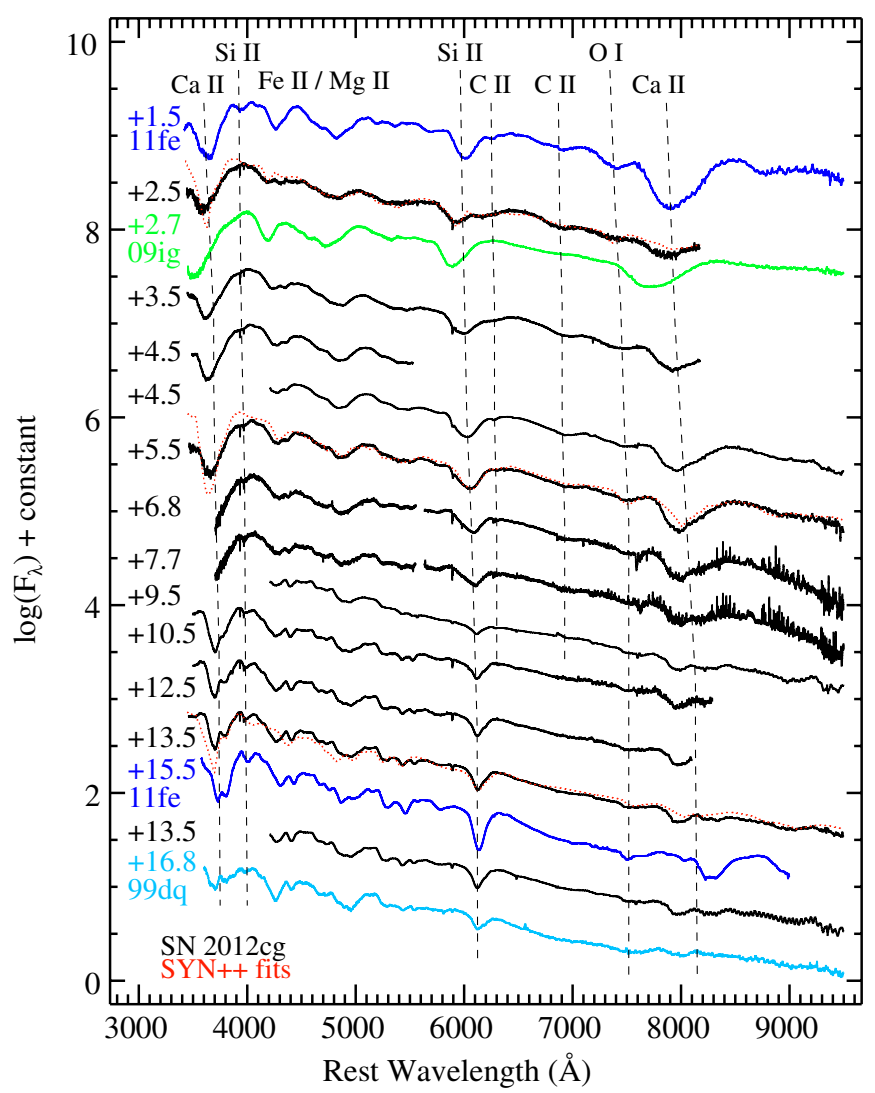

Figure 3. Spectra of SN 2012cg (black) and some SYN++ fits (red), along with comparisons to other young SNe Ia: SNe 2011fe (dark blue; Parrent et al. 2012), 2009ig (green; Foley et al. 2012), and 1999dq (light blue; Silverman et al. 2012a). Each spectrum is labeled with its age relative to explosion. The data have all been deredshifted and dereddened. Major spectral features are identified. The noise at the red end in the $\sim 7$ and $\sim 8$ day spectra is due to incomplete removal of night-sky emission lines.

$\mathrm{H}$ and $\mathrm{K}$ absorption from the host galaxy, though we usually do not resolve the former. The median redshift as determined from both of these features is $z=0.00152 \pm 0.00024$, consistent with the published redshift of NGC 4424 ( $z=0.00146$; Kent et al. 2008).

The equivalent width (EW) of $\mathrm{NaI} \mathrm{D}$ absorption is often converted into reddening, but there is some controversy over the exact relationship (Poznanski et al. 2011, and references therein). No Na I D absorption is detected at $z=0$; thus, we use $E(B-V)_{\mathrm{MW}}=0.019 \mathrm{mag}$, as given by Schlegel et al. (1998). The median EW of Na I D from the host galaxy is measured to be $\sim 0.7 \AA$, which yields a range of possible reddening values (0.00-0.60 mag) with a nominal value of $0.22 \mathrm{mag}$ (with the majority of the uncertainty coming from the scatter in the relationship presented in Poznanski et al. 2011). This is consistent with $E(B-V)_{\text {host }} \approx 0.18 \mathrm{mag}$, the value derived from the photometry in Section 4.1.

\subsection{1. $S Y N++$}

To help identify the species present in our spectra of SN 2012cg, we used the spectrum-synthesis code SYN++ (Thomas et al. 2011). SYN++ is derived from SYNOW (Fisher et al. 1997) and is a parameterized resonance-scattering code that allows for the adjustment of chemical composition, optical depths, temperatures, and velocities. Three examples of our SYN++ fits are shown in Figure 3.

Our first spectrum of SN 2012cg (2.5 days after explosion) consists of absorption features from ions usually seen in $\mathrm{SNe}$
Ia (Ca II, Si II, Fe II, Mg II, S II, and O I), as well as C II which is found in over one-fourth of all SNe Ia (e.g., Silverman \& Filippenko 2012). All of these species have expansion velocities $\gtrsim 14,000 \mathrm{~km} \mathrm{~s}^{-1}$, similar to what was found in the earliest spectra of SN 2011fe (Parrent et al. 2012) and SN 2009ig (Foley et al. 2012). The $C$ II features $\lambda 6580$ and $\lambda 7234$ are detected in our first spectrum, become more distinct but weaken with time, and by 10.5 days past explosion the only sign of $\mathrm{C}$ is a flattening of the red wing of the Si II $\lambda 6355$ feature (presumably due to $\mathrm{C}_{\text {II }} \lambda$ 6580). Just 2 days later no $\mathrm{C}_{\mathrm{II}}$ is required in our SYN++ fits.

In addition to the usual photospheric Si II absorption, SN 2012cg exhibits high-velocity (HV) Si II $\lambda 6355$ in its earliest spectra. This HV absorption appears to be detached from the rest of the photosphere, with our SYN++ fits showing a gap of $\sim 4000 \mathrm{~km} \mathrm{~s}^{-1}$ separating the normal and HV Si II $\lambda 6355$ features. The HV feature dominates the Si II $\lambda 6355$ profile in our first spectrum (also seen by Marion et al. 2012), but only 1 day later the normal and HV features are roughly equal in strength. By $\sim 7$ days past explosion, the normal-velocity feature dominates the Si II $\lambda 6355$ profile and our SYN++ fits to spectra older than 12.5 days past explosion do not require any HV Si II. This temporal evolution of the HV Si II $\lambda 6355$ feature almost exactly mirrors that of SN 2009ig (Foley et al. 2012).

Similarly, Ca II also displays HV features in our first spectrum. Both the $\mathrm{Ca}$ II $\mathrm{H}$ and $\mathrm{K}$ feature and the $\mathrm{Ca}$ II near-IR triplet show absorption profiles that consist of a photospheric component as well as an HV component with a velocity gap of $4000-8000 \mathrm{~km} \mathrm{~s}^{-1}$. In contrast to the HV Si II, the HV Ca II is required in all of our SYN++ fits, through 13.5 days past explosion. HV Ca II was also seen in spectra of SN $2011 \mathrm{fe}$ younger than $\sim 8$ days past explosion (Parrent et al. 2012).

\subsubsection{Individual Line Measurements}

To more precisely study the evolution of our spectra of SN 2012cg, we directly measured the expansion velocities and EWs of some of the absorption features; see Silverman et al. (2012b) for details. All of the measured velocities are consistent with our SYN++ fits and are plotted in Figure 4.

As mentioned previously, all of our SN $2012 \mathrm{cg}$ spectra show evidence for $\mathrm{HV}$ Ca II. In our first spectrum, 2.5 days past explosion, the $\mathrm{Ca}$ II $\mathrm{H}$ and $\mathrm{K}$ feature and $\mathrm{Ca}$ II near-IR triplet have velocities of $\sim 30,000 \mathrm{~km} \mathrm{~s}^{-1}$, which is even higher than in the earliest spectra of SN $2011 \mathrm{fe}$ (Parrent et al. 2012). As seen in the top panel of Figure 4, the two Ca II features have very similar velocity evolution in both the $\mathrm{HV}$ and photospheric components.

Also plotted in the top panel of Figure 4 is the velocity evolution of the $\mathrm{O}$ I triplet. Initially, these velocities are relatively large and similar to the photospheric O I velocity seen in the earliest spectra of SN 2011fe. However, that SN also showed $\mathrm{HV}$ O I earlier than 5 days after explosion which is absent from SN 2012cg (Parrent et al. 2012).

As discussed in Section 4.2.1 and seen in the bottom panel of Figure 4, SN 2012cg exhibited an HV Si II $\lambda 6355$ feature in the earliest observations. Our spectrum from 2.5 days past explosion shows the HV Si II $\lambda 6355$ to be at $\sim 22,000 \mathrm{~km} \mathrm{~s}^{-1}$, which is slightly lower than the HV Si II seen in either SN 2009ig (Foley et al. 2012) or SN 2011fe (Parrent et al. 2012). If we associate the steepening of the red edge of the Ca II H and $\mathrm{K}$ feature in our earliest spectra (which was also seen in SN 2009ig; Foley et al. 2012) with absorption from Si II $\lambda 4000$, the velocity of this feature is consistent with that of the Si II $\lambda 6355$ line. 

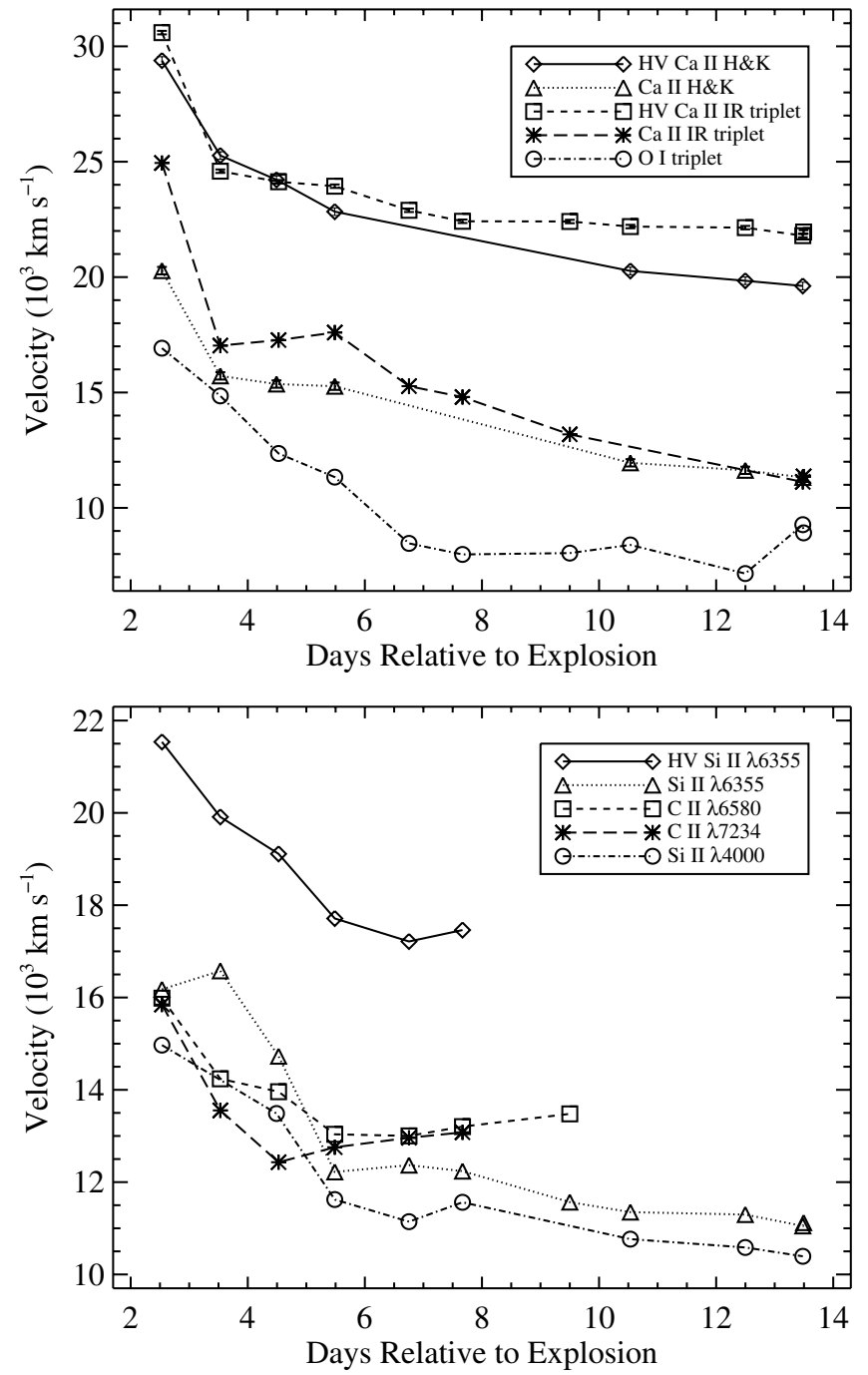

Figure 4. Temporal evolution of the expansion velocity of Ca II (both HV and photospheric components) and O I features (top) and of Si II (both HV and photospheric components) and C II features (bottom). Uncertainties are smaller than the size of the data points.

Also in the bottom panel of Figure 4, we plot the velocity evolution of the $\mathrm{C}$ II features $\lambda 6580$ and $\lambda 7234$. The velocity of $\mathrm{C}_{\text {II }} \lambda 7234$ is slightly lower than that of $\mathrm{C}_{\text {II }} \lambda 6580$ and quite similar to that of the photospheric component of Si II $\lambda 6355$, which has been seen in previous work (e.g., Silverman \& Filippenko 2012). The ratio of the velocity of $C_{\text {II }} \lambda 6580$ to Si II $\lambda 6355$ decreases slightly with time, though in each spectrum this ratio is within $10 \%$ of the median value found for a large sample of SNe Ia (e.g., 1.05; Silverman \& Filippenko 2012). We also measure the EW of both of the $C_{\text {II lines and find }}$ that there is some evidence for a factor of 2-3 increase in the EW of $\mathrm{C}$ II $\lambda 6580$ from 5.5 to 9.5 days past explosion, but this is significantly smaller than the factor of $\sim 5$ increase at similar epochs observed in one object (SN 1994D; Silverman \& Filippenko 2012) and predicted from synthetic spectra (Folatelli et al. 2012).

\subsubsection{Classification}

Using the SuperNova IDentification (SNID) code (Blondin \& Tonry 2007) along with the spectral templates described by Silverman et al. (2012a), we find that SN 2012cg is spectroscopically similar to the somewhat peculiar SN 1999aa and its ilk (Li et al. 2001; Strolger et al. 2002; Garavini et al. 2004). SNID indicates that several of the oldest spectra presented here are most similar to those of SN 1999aa-like objects (at the correct redshift and age). Branch et al. (2006) utilized the near-maximum brightness EWs of Si II $\lambda 6355$ and Si II $\lambda 5972$ in order to classify SN Ia spectra. Using the oldest spectrum presented in this work, we find that both of these features are weak and thus SN 2012cg definitely falls in the shallow silicon class (as do SN 1999aa and its brethren).

The oldest spectrum of SN 2012cg presented here (from 13.5 days after explosion) vaguely resembles both $\mathrm{SNe} 2009 \mathrm{ig}$ (not shown) and 2011fe at similar epochs. However, SN 2012cg has significantly weaker Si II absorption and still shows HV Ca II. As seen at the bottom of Figure 3, a much better match to this spectrum of SN 2012cg is the SN 1999aa-like SN 1999dq from $\sim 17$ days past explosion (Silverman et al. 2012a; Ganeshalingam et al. 2011). Thus, our spectral data indicate that SN 2012cg is an SN 1999aa-like object. This, along with the fact that SN $2012 \mathrm{cg}$ is overluminous at maximum brightness (Section 4.1), suggests that it will have a slower-than-average light-curve decline. ${ }^{12}$

\section{CONCLUSIONS}

In this Letter we presented optical photometry and spectroscopy of SN 2012cg, an SN Ia discovered a mere $1.5 \pm 0.2$ days after explosion. It is found to be overluminous at maximum brightness and spectroscopically similar to the somewhat peculiar SN Ia 1999aa. These properties imply that SN 2012cg will have a slowly declining light curve. However, the pre-maximumbrightness photometry indicates a faster-than-average rising light curve. Future observations will be required to definitively determine the relative normalcy (or peculiarity) of SN 2012cg.

This object will certainly become one of the best-studied SNe Ia ever. In under 3 years there have been three very nearby $\mathrm{SNe}$ Ia discovered within days after explosion (SNe 2009ig, 2011fe, and $2012 \mathrm{cg}$ ). During that time transient surveys of various sizes and cadences have continued to grow and evolve, and yet these extremely young SNe Ia are still rare finds. Therefore, they will serve as excellent case studies well into the future and will help us answer some of the many outstanding questions in the field of SNe Ia that plague us today.

We thank J. Caldwell, J. R. Mould, S. Odewahn, J. X. Prochaska, S. Rostopchin, M. Shetrone, and I. Shivvers for their assistance with some of the observations. We are grateful to the staff at Lick Observatory for their support and are indebted to the benefactors, builders, and partners of the HET and LRS. This work was supported in part by NSF grant AST-1109801 and Hungarian grant OTKA K76816. A.V.F.'s group at UC Berkeley, and KAIT and its ongoing operation, have received financial assistance from the Sylvia \& Jim Katzman Foundation, Gary \& Cynthia Bengier, the Richard \& Rhoda Goldman Fund, the TABASGO Foundation, and NSF grant AST-0908886.

\section{REFERENCES}

Blondin, S., \& Tonry, J. L. 2007, ApJ, 666, 1024

Bloom, J. S., Kasen, D., Shen, K. J., et al. 2012, ApJ, 744, L17

Branch, D., Dang, L. C., Hall, N., et al. 2006, PASP, 118, 560

Cenko, S. B., Filippenko, A. V., Silverman, J. M., et al. 2012, ATel, 4115, 1 Chevalier, R. A. 1992, ApJ, 394, 599

12 Post-maximum photometric data indicate $\Delta m_{15} \approx 0.83 \mathrm{mag}$, which is indeed slower than average. 
Conley, A., Guy, J., Sullivan, M., et al. 2011, ApJS, 192, 1

Cortés, J. R., Kenney, J. D. P., \& Hardy, E. 2006, AJ, 131, 747

Cortés, J. R., Kenney, J. D. P., \& Hardy, E. 2008, ApJ, 683, 78

Cortini, G. 2012, CBET, 3111, 1

Dopita, M., Hart, J., McGregor, P., et al. 2007, Ap\&SS, 310, 255

Filippenko, A. V., Li, W. D., Treffers, R. R., \& Modjaz, M. 2001, in ASP Conf. Ser. 246, Small Telescope Astronomy on Global Scales, ed. B. Paczyński, W. P. Chen, \& C. Lemme (San Francisco, CA: ASP), 121

Fisher, A., Branch, D., Nugent, P., \& Baron, E. 1997, ApJ, 481, L89

Folatelli, G., Phillips, M. M., Morrell, N., et al. 2012, ApJ, 745, 74

Foley, R. J., Challis, P. J., Filippenko, A. V., et al. 2012, ApJ, 744, 38

Ganeshalingam, M., Li, W., \& Filippenko, A. V. 2011, MNRAS, 416, 2607

Ganeshalingam, M., Li, W., Filippenko, A. V., et al. 2010, ApJS, 190, 418

Garavini, G., Folatelli, G., Goobar, A., et al. 2004, AJ, 128, 387

Hill, G. J., Nicklas, H. E., MacQueen, P. J., et al. 1998, Proc. SPIE, 3355, 375

Horesh, A., Kulkarni, S. R., Fox, D. B., et al. 2012, ApJ, 746, 21

Howell, D. A. 2011, Nature Commun. 2

Jha, S., Kirshner, R. P., Challis, P., et al. 2006, AJ, 131, 527

Jordi, K., Grebel, E. K., \& Ammon, K. 2006, A\&A, 460, 339

Kasen, D. 2010, ApJ, 708, 1025

Kent, B. R., Giovanelli, R., Haynes, M. P., et al. 2008, AJ, 136, 713

Kleiser, I., Cenko, S. B., Li, W., \& Filippenko, A. V. 2009, CBET, 1918, 1

Landolt, A. U. 1992, AJ, 104, 340

Li, W., Filippenko, A. V., Treffers, R. R., et al. 2001, ApJ, 546, 734

Lipunov, V., \& Krushinsky, V. 2012, ATel, 4138, 1

Marion, G. H., Kirshner, R. P., Foley, R. J., \& Challis, P. 2012, CBET, 3111,3
Matheson, T., Filippenko, A. V., Ho, L. C., Barth, A. J., \& Leonard, D. C. 2000, AJ, 120, 1499

Miller, J. S., \& Stone, R. P. S. 1993, Lick Observatory Technical Report 66 (Santa Cruz, CA: Lick Observatory)

Nugent, P., Sullivan, M., Bersier, D., et al. 2011a, ATel, 3581, 1

Nugent, P. E., Sullivan, M., Cenko, S. B., et al. 2011b, Nature, 480, 344

Parrent, J. T., Howell, D. A., Friesen, B., et al. 2012, ApJ, 752, L26

Perlmutter, S., Aldering, G., Goldhaber, G., et al. 1999, ApJ, 517, 565

Poznanski, D., Ganeshalingam, M., Silverman, J. M., \& Filippenko, A. V. 2011, MNRAS, 415, L81

Rabinak, I., Livne, E., \& Waxman, E. 2011, arXiv:1108.5548

Richmond, M., Treffers, R. R., \& Filippenko, A. V. 1993, PASP, 105, 1164

Riess, A. G., Filippenko, A. V., Challis, P., et al. 1998, AJ, 116, 1009

Riess, A. G., Filippenko, A. V., Li, W., \& Schmidt, B. P. 1999, AJ, 118, 2668

Röpke, F. K., Kromer, M., Seitenzahl, I. R., et al. 2012, ApJ, 750, L19

Schlegel, D. J., Finkbeiner, D. P., \& Davis, M. 1998, ApJ, 500, 525

Silverman, J. M., \& Filippenko, A. V. 2012, MNRAS, in press (arXiv:1202.3788)

Silverman, J. M., Foley, R. J., Filippenko, A. V., et al. 2012a, MNRAS, in press (arXiv:1202.2128)

Silverman, J. M., Kong, J. J., \& Filippenko, A. V. 2012b, MNRAS, in press (arXiv:1202.2129)

Strolger, L., Smith, R. C., Suntzeff, N. B., et al. 2002, AJ, 124, 2905

Suzuki, N., Rubin, D., Lidman, C., et al. 2012, ApJ, 746, 85

Thomas, R. C., Nugent, P. E., \& Meza, J. C. 2011, PASP, 123, 237

Wade, R. A., \& Horne, K. 1988, ApJ, 324, 411

Wang, X., Li, W., Filippenko, A. V., et al. 2009, ApJ, 697, 380 